Methods To determine the biological age, the method of V.P. Voitenko, in which the indicator of biological age is compared with the value of the proper biological age, calculated by the formula using the calendar age of the subject. The number of respondents was 300 workers of mines of the following professions: 80 tunnellers, 50 drillers, 50 electrical fitters, 100 miners and 20 foremen.

Results The average biological age of workers, equal to 46.6 years, exceeded the proper biological age by 6 years (with an average work experience of 14.2 years) and was more than 10 years higher than the calendar age. This means that the aging of miners was more pronounced in comparison with the population standard of aging. It should be noted that the differences between the values of the calendar age and the proper biological age in miners increased with an increase in the calendar growth, which indicates that the rate of aging in miners was most pronounced in middle and older age.

Conclusion The average biological age of miners, equal to 45.6 years, exceeded the proper biological age by 5 years (with an average work experience of 14.5 years) and was 9 years higher than their calendar age.

\section{P-454 LOW BACK PAIN IN THE CARE SETTING: STUDY AMONG 300 HEALTHCARE STAFF FROM TWO UNIVERSITY HOSPITAL IN TUNISIA}

${ }^{1}$ Kamoun Salma, Olfa Jlassi, Lamia Bouzgarrou, Neila Chaari, Adel Amri, Irtyah Merchaoui. ${ }^{1}$ Centre Militaire de Médecine du Travail et de Sécurité Professionnelle, Tunisia

\subsection{6/OEM-2021-EPI.335}

Introduction Low back pain (LBP) is one of the leading musculoskeletal disorders and it is a disabling occupational hazard. It is also a common cause of morbidity among the healthcare staff within were more vulnerable to LBP.

Objectives This study aimed to assess prevalence of LBP among healthcare staff and identify the socio-occupational hazards of this morbidity.

Methods This is a cross-sectional study, carried out over 14 months, with 300 healthcare staff from two university hospitals in Tunisian center. It is based on an analysis of sociooccupational profile, the assesement of the Work Ability Index (WAI), the Nordic musculoskeletal questionnaire as well as assessment tests of physical condition and flexibility of the spine (hand-floor distance; sit-stand test; balance test)

Results The sex ratio of the sample was 1.06, the average age was $42.64 \pm 11.65 .20 \%$ of the healthcare staff in the sample had a BMI larger than 30 and $51.9 \%$ of them did not practice any regular sports activity. The perceived physical workload was considered " heavy " by $41.6 \%$ of the staff questioned. $75 \%$ of the healthcare staff in the sample had a good to excellent work ability according to the WAI. Over the 12 months preceding the survey, two third of the healthcare staff in the sample complained of LBP, with pain assessed as 'severe' or 'extremely severe' in $54.4 \%$ of cases. The LBP was statistically correlated with female gender $(p=0.01)$ and with impaired work ability $\left(p<10^{-3}\right)$. The average finger-ground distance was $7.10 \pm 7.5 \mathrm{~cm}$ and it was statistically correlated with LBP $(p=0.05)$, with age $\left(\mathrm{p}<10^{-3}\right)$, with seniority $\left(\mathrm{p}<10^{-3}\right)$ and with a BMI $\geq 25$ $\left(\mathrm{p}=10^{-3}\right)$.

Conclusion LBP persists as a real health problem among healthcare staff with heavy professional and social repercussions. Reducing their prevalence and impact is based on improving working conditions and multidisciplinary care.

\section{P-455 ASSESSING QUALITY OF LIFE, PRODUCTIVITY LOSS AND ACTIVITY IMPAIRMENT IN ASTHMA}

${ }^{1}$ Amira Omrane, Olfa Jlassi, Raja Romdhani, Nadia Boudaouara, Imen Touil, Soumaya Bouchareb, Lamia Bouzgarrou, Taoufik Khalfallah, Leila Boussoffara, Jalel Knani. ${ }^{1}$ Faculty of Medicine of Monastir, Tunisia

\subsection{6/OEM-2021-EPI.336}

Background Asthma can be associated with substantial quality of life (QoL) alteration, productivity loss and activity impairment. We sought to assess the quality of life and the performance characteristics of an asthma-specific adaptation of the Work Productivity and Activity Impairment Questionnaire (WPAI:Asthma) in Tunisian young and active patients with asthma

Patients and Methods A prospective study was carried out during a year in active asthmatic patients, aged less than 65 years and consulting in the pulmonology department of a public hospital in central region of Tunisia. The WPAI:Asthma was administered to assess productivity loss. Asthma Quality-of-Life Questionnaire (AQLQ) in its validated arabic version was used to assess the QoL.

Results One hundred and one patients were enrolled in this study. Patients had a mean age of $44.2 \pm 13.2$ years and were predominantly female (66.3\%). The mean job tenure was 7.3 \pm 7.7 years. Workers belonged to the textile sector in $29.3 \%$ of cases. Asthma was allergic in $84.2 \%$ of cases and occupational in 18.2\%. Asthma was uncontrolled in $62.4 \%$ and worsened at work in $71.3 \%$. The mean AQLQ score was $5.08 \pm 1.2$. This score was statistically correlated with the occupational etiology $(p=0.001)$, the worsening of asthma symptoms at work $(p=0.016)$, the absence of professional reclassification $(p=0.002)$, the severity asthmatic disease $(p=$ $0.001)$ and uncontrolled asthma $(p=0.001)$. Absenteeism was $4.2 \pm 14.6 \%$ while presenteeism was $33.1 \pm 25.9 \%$. The resulting loss of productivity was $30.4 \pm 22.2 \%$. Activity impairment was statistically correlated with female gender $(p=0.02)$, alcohol consumption $(p=0.001)$, occupational etiology $(p=0.03)$ and uncontrolled asthma $(\mathrm{p}=0.001)$.

Conclusion This study revealed significant levels of activity impairment and QoL alteration among active and young patients suffering from asthma. Improving asthma control, working condition and mental health may be important targets for enhancing workplace productivity in asthma. Presenteeism, absenteeism and productivity loss may represent key metrics to assess intervention effectiveness in active and young patients.

\section{P-457 WORK ORGANIZATION AS A PREVENTIVE FACTOR OF WORK-RELATED LOW BACK PAIN IN TUNISIAN CRAFTSMEN}

${ }^{1}$ Asma Kheder, Ben Afia Latifa, Meriem Heni, Amira Omrane, Olfa Jlassi, Nesrine Mars, Harrathi Chayma, Taoufik Khalfallah, Lamia Bouzgarrou. 'University of Monastir - School of Medicine, Tunisia

\subsection{6/OEM-2021-EPI.337}

Introduction Work-related low back pain (WRLBP) is a complex phenomenon accounting for a high proportion of 
occupational costs. Growing evidence links organizational risk factors to WRLBP.

Objective To examine the impact of work organization on WRLBP and mental health of Tunisian craftsmen.

Methods A cross-sectional study was conducted among the registered craftsmen of the governorate of Monastir (Tunisia) $(n=8526)$. The research was implemented on a representative sample, stratified by gender and craft activity. The survey included items related to health status, work organization, in addition to the Nordic Musculoskeletal and the Job Content Questionnaires.

Results The sample consisted of 368 craftsmen with a mean age of $42.7 \pm 8.5$ years and a sex-ratio of 0.88 . Work schedules were fixed with a mean duration of $8 \pm 2$ hours. Craftsmen had at least one break per day with a mean duration of $60 \pm 12$ minutes. Freedom in item design was found in $39 \%$ of cases. Room for manoeuvre was acceptable in 53\% of cases. Mutual help was possible in $40 \%$ of cases. Relationships with colleagues and superiors were 'good' in more than $80 \%$ of cases. According to the Karazek model, 13.6\% were in a Job Strain situation. WRLBP during the week preceding the survey were reported by $0.8 \%$ of the workers and $14.9 \%$ of them reported WRLBP during the last 12 months. Statistical analysis showed that WRLBP were less frequently reported by craftsmen with fewer hours of work $\left(\mathrm{p}<10^{-3}\right)$, those who had more than one break per day $\left(\mathrm{p}<10^{-3}\right)$ and the freedom of item design with non-monotonous production $\left(\mathrm{p}<10^{-3}\right)$. WRLBP was also less reported by workers non-constrained by an accelerated pace of work $\left(\mathrm{p}<10^{-3}\right)$, by a strict monitoring of work $(p=0.008)$, or by an increased sense of responsibility $\left(\mathrm{p}<10^{-3}\right)$.

Conclusion The risk factors for WRLBP are complex. Our research suggests that the role of organizational and psychosocial factors need to be better examined.

\section{P-458 GENDER INFLUENCE OF THE CLINICAL EXPRESSION AND SEVERITY OF COVID-19 INFECTION AMONG HEALTH CARE WORKERS}

${ }^{1}$ Lamia Bouzgarrou, Amen Moussa, Ben Afia Latifa, Amira Omrane, Safa Hsen, Touka Yahyaoui, Harrathi Chayma, Asma Kheder, Taoufik Khalfallah, Jihene Chili. ${ }^{1}$ University of Monastir, Tunisia

\subsection{6/OEM-2021-EPI.338}

Introduction Since the start of the COVID-19 epidemic, hospital staff have been massively affected in most countries, including Tunisia.

Objective This study aimed to examine the influence of the gender dimension on COVID-19 contamination expression among Tunisian hospital staff.

Methods Based on the COVID-19 register kept by the occupational medicine service and dedicated to hospital staff, data over 8 months were analyzed (September 2020-April 2021). COVID-19 screening was performed among all symptomatic hospital employees, or asymptomatic ones identified as close contact of a patient, colleague or relative confirmed positive for COVID-19. A nasypharyngeal swab was used for viral testing (reverse transcription polymerase chain reaction (RTPCR) and SARS-CoV-2 Rapid Antigen Test). Chest tomography was reserved for suspected cases with negative viral tests.
Results In total, 419 COVID-19 infections were confirmed by rt-PCR in $63.96 \%$, by rapid antigen testing in $33.65 \%$ and tomography in $2.39 \%$ of cases. Women represented $72.21 \%$ of contaminated staff, with average age of $21.75 \pm 9.78$ years but with no difference between gender groups. The department most affected was gynecology (43 cases), and nurses were the most affected category, especially among female staff $(p=0.016)$. In addition, fever $(p=0.012)$, neurological symptoms $(\mathrm{p}=0.049)$, such as aguesia $(\mathrm{p}=0.003)$, were more common in female workers. In contrast, digestive symptoms were more frequent in men $(p=0.0049)$, especially diarrhea $(p=0.00)$. In addition, chest tomography COVID-19 confirmation was significantly more common in men $(\mathrm{p}=0.012)$. Hospitalization was indicated in 16 cases, in intensive care in 2 cases with one case of death. No significant difference was noted between genders based on the severity of the COVID19 infection.

Conclusion Gender clinical difference of COVID-19 clinical expression was suggested by our results among health workers without impact on severity. These observations should be confirmed in larger studies.

\section{P-459 COPING STRATEGIES OF HOSPITAL NURSES IN THE COVID-19 PANDEMIC CONTEXT}

'Rosângela Aparecida de Sousa, Vivian Mininel. 'Universidade Federal de São Carlos, Brazil

\subsection{6/OEM-2021-EPI.339}

Introduction Occupational stress is one of the negative factors present in nursing work, especially in the hospital context; that factor was exasperated by the Covid-19 pandemic. Organizational strategies are very useful to reduce stress at work, individual coping behaviors can also contribute to the understanding of this factor.

Objective This study aims to understand coping strategies of hospital nurses with occupational stress in the Covid-19 pandemic context.

Method This is a qualitative, descriptive and exploratory study, which was developed at a university hospital located in the state of São Paulo, Brazil. The target population was male and female nurses who were employed in the research hospital. Data were collected through virtual individual interviews, based on a previously defined semi-structured questionnaire, and analyzed according to thematic content analysis. Intentional sampling was used and the participants were recruited until data saturation. The project was approved by study scenario and Ethics Committee for Research with Human Beings at Federal University of São Carlos; all ethical precepts were observed.

Partial Results Thirteen nurses participated in the data collection: 10 (76.92\%) women and $3(23.07 \%)$ men, aged between 31 and 55 years old. Working as a nurse: 1 (7.69\%) worked between 1 to 5 years, $3(23.07 \%)$ between 5.1 to 10 years, 6 (46.15\%) between 10.1 to 20 years and $3(23.07 \%)>20$ years. For the duration of work experience at the studied institution: $1(7.69 \%)<1$ year, $9(69.23 \%)$ between 1 to 5 years and $3(23.07 \%)$ between 5.1 to 10 years. It was expected, with the results of this research, to understand the coping strategies used by hospital nurses to cope with occupational stress in the current pandemic scenario of COVID-19. 